TITRE: QuAND DÉCLARER, C'EST FAIRE UNE IDENTITÉ. VERS UNE ONTOLOGIE DE L'IDENTITÉ DISCURSIVE À TRAVERS DES LETTRES DE TUEURS EN SÉRIE

Auteur(s): Abdelhadi BellachHab, Maître de confÉrences HDR, CoDiRe, EA 4643, Université de NANTES

PUBLICATION: ÉCRITS HORS-NORMES

PAGES: $115-126$

Directeurs: Agnès Steuckardt et Karine Collette

ÉdITEUR: LES ÉDITIONS DE L'UNIVERSITÉ DE SHERBROOKE, 2019

ISBN: 978-2-7622-0360-8

URI: HTTP://HDL.HANDLE.NET/11143/15576

DOI: HTTPS://DOI.ORG/10.17118/11143/15576 


\section{Quand déclarer, c'est faire une identité. Vers une ontologie de l'identité discursive à travers des lettres de tueurs en série}

Abdelhadi Bellachhab, Maître de conférences HDR CoDiRe, EA 4643, Université de Nantes

Résumé : Cette étude porte sur les lettres envoyées par Robin Gecht, tueur en série condamné à mort, à Jennifer Furio. Dans le prolongement de la contribution d'Olga Galatanu au présent ouvrage, elle se situe dans le cadre théorique de la Sémantique des Possibles Argumentatifs et analyse les mécanismes sémantico-discursifs d'une dynamique de restauration identitaire. Pour régénérer son image, Robin Gecht joue sur les valeurs épistémiques, morales, déontiques et axiologiques. Ses lettres constituent des actes de langage de type déclaratif, qui revendiquent le statut de « personne normale » par opposition au statut de personne « anormale » ou « hors-normes » que les déclarations judiciaires et médiatiques lui ont attribué.

Mots-clés : Sémantique des Possibles Argumentatifs, cinétisme sémantique, reconstruction discursive de soi, dynamique de restauration identitaire, espace épistolaire.

Abstract: This study focuses on letters sent by Robin Gecht, serial killer sentenced to death, to Jennifer Furio. As an extension of Olga Galatanu's contribution further in this book, she is situated in the theoretical frame of the Semantics of Argumentative Possibilities and analyzes the semantico-discursive mechanisms of a dynamic of identity restoration. To regenerate his image, Robin Gecht plays on epistemic, moral, deontic and axiological values. His letters are declarative speech acts, claiming the status of « normal person » opposed to « abnormal » or « non-standard » status that legal and media declaratory acts have attribued him.

Key words: Semantics of Argumentative Potentials, semantic kinesis, discursive reconstruction of oneself, identity restoration dynamics, epistolary space. 
Qu'ils soient marginaux, atypiques ou non-conventionnels, les discours hors-normes se démarquent par une forme d'affranchissement d'une sorte d'institutionnalisation, normative par définition, et bouleversent de fait les normes discursives qui sous-tendent les discours normés et normatifs. Cette anormativité discursive se veut, selon nous, une recréation d'une réalité sociale où la reconnaissance institutionnalisée n'est pas envisagée et où la réalité hors-normes, reconstruite, se revendique norme. Comment peut-on donc saisir l'anormativité de discours hors-normes tels que les lettres de tueurs en série, ces derniers se trouvant dans une logique de reconstruction et de « restauration identitaire » (Barbier \& Galatanu, 1998: 62) pour soi et pour autrui ? C'est la réponse à cette question qui constituera l'objectif premier de notre article, lequel s'inscrit dans la suite de l'article de Galatanu (ici même).

Nous cherchons à saisir la normativité revendiquée au travers d'une anormativité discursive apparente, voire attestée, où une identité criminelle se reconstruit à partir d'une antinomie visible, à savoir celle de réduire l'écart entre une identité criminelle et une autre « saine » (normale) et, en même temps, s'écarter de l'anormativité des autres criminels.

Les lettres de tueurs en série, du moins par leur singularité ontologique, pourraient avoir une existence "pseudo-objective », dans la mesure où elles semblent échapper à une catégorisation subjective; elles sont en fait, comme toute réalité discursive et sociale, dépendantes de l'observateur, c'est-à-dire qu'elles n'ont aucune existence en dehors de toute reconnaissance institutionnelle au sens d'une reconnaissance par l'autre. Bref, ces lettres de tueurs en série, avec ce qu'elles proposent comme reconstruction identitaire, ont une ontologie subjective, qui a besoin d'une institution, de l'autre, représenté par Jennifer Furio (la correspondante de ces tueurs en série) et, à travers elle, ces lecteurs, pour définir leur existence.

Afin d'examiner l'identité discursive des tueurs en série, condamnés aux États Unis, nous proposons d'étudier les onze lettres de Robin Gecht (Furio, 1998: 147-174), un tueur en série condamné à 120 ans de prison, et membre du groupe du « Chicago rippers » (Éventreurs de Chicago), composé de trois autres membres dont Edward Spreitzer, faisant l'objet d'étude de Galatanu (ici même). Les lettres de Robin Gecht ont été envoyées, entre juin 1997 et avril 1998, à Jennifer Furio, une correspondante qui dit, dans sa lettre envoyée à environ une cinquantaine d'incarcérés, vouloir comprendre ce qui s'est passé du point de vue de ces condamnés et comment ils se retrouvent en prison (Furio, 1998: VII-VIII).

Après une inscription épistémologique, qui rejoint le double ancrage théorique et conceptuel adopté par Galatanu (ici même), mobilisant la Sémantique des Possibles Argumentatifs au service des identités et leurs dynamiques, nous tentons de reconstruire, à travers une analyse linguistique du discours, l'univers sémantico-discursif de Robin Gecht, d'abord du point de vue de l'acte criminel au travers d'opérations énonciatives diverses, notamment de dénomination, et ensuite du point de vue de l'acteur et de son image.

\section{Vers une ontologie de la réalité discursive}

En complémentarité avec le cadrage théorique développé dans l'article de Galatanu (ici même), nous situons notre contribution dans la perspective d'une ontologie sociale, en l'occurrence celle développée par Searle depuis 1995, où « la réalité institutionnelle dans son ensemble est créée par un certain type d'acte de langage, de représentation linguistique, qui permet aussi de pérenniser son existence » (Searle, dans Monnet, Navarro, 2009). Nous présumons que cette conception pourrait s'étendre à la création de la réalité discursive, où le discours est une création ou une recréation de la réalité sociale. 
Dans cette ontologie sociale, nous dit Searle (2010), c'est grâce aux Déclarations - comme acte de langage - que nous faisons exister quelque chose en représentant seulement le fait qu'il existe. Ces Déclarations créent des «fonctions-statuts ». Ainsi la revendication d'identité génère une fonction-statut : la fonction-statut d'identité est à un individu ce que le statut d'argent est à un billet de 10 euros, par exemple. La fonction-statut d'identité équivaut à la fonction-statut d'argent. Elles imposent toutes les deux une ontologie subjective. De ce fait:

« la réalité institutionnelle est maintenue dans le temps grâce à des représentations qui ont la même forme logique que les Déclarations de fonction-statut, même si ni leur création, ni leur pérennisation ne nécessitent la forme syntactique explicite d'un acte de parole déclaratif. C'est simplement en représentant leur existence que l'on fait exister des fonctions-statuts. C'est ainsi que fonctionnent la création et le maintien de l'ensemble de la réalité institutionnelle et, partant, de la civilisation humaine » (Searle, dans Monnet, Navarro, 2009).

Revendiquer donc une identité, en se distinguant des trois autres membres de la bande de « Chicago Rippers », à travers des correspondances, c'est « déclarer » une identité, et, comme on va le voir, une identité «normale ». Robin Gecht cherche en effet à se régénérer, à s'attribuer une valeur, une « fonction-statut » autre que celle de criminel, ou de tueur en série. Inculpé de tentative d’homicide et condamné à la réclusion criminelle, il déclare à travers ses lettres adressées à Jennifer Furio qu'il a une identité autre que celle qu'on - le système médiatique et judiciaire en l'occurrence - lui a attribuée, laquelle est une identité horsnormes, anormale. Et comme toute identité qui a besoin de s'exprimer à travers le langage, quoi de mieux que de le faire à travers des déclarations sous forme de lettres, lui permettant de se faire reconnaître une identité, par l'observateur, par l'institution représentée par la correspondante, Jennifer Furio, symbolisant le monde extérieur. Robin Gecht cherche par le biais de son discours à se reconstruire ou à restaurer une image normale en lui donnant une existence, quoique subjective parce qu'elle est dépendante de l'observateur, Jennifer Furio, mais qui lui octroie une réalité, une reconnaissance, donc une ontologie. Galatanu affirme, à cet égard, que :

" les enjeux de l'échange sont pour les sujets incarcérés directement et fondamentalement identitaires. S'ils acceptent de participer [...], c'est pour pouvoir justement communiquer des images d'eux-mêmes, a priori différentes des images qu'ils pensent ou qu'ils savent que les autres ont et proposent d'eux: "des sujets coupables, jugés et condamnés à des peines très lourdes "» (2010: 127).

De ce fait, la construction identitaire dans le discours hors-normes de ce tueur en série se ferait à partir d'une restauration qui cherche à renouveler l'identité hors-normes dessinée à travers les médias, reconnue socialement et institutionnellement à partir du moment où il est condamné.

En lien avec cette ontologie sociale, la perspective sémantico-discursive proposée par la Sémantique des possibles argumentatifs quant à sa définition de l'identité « comme une construction mentale et discursive de soi et d'autrui, dans des contextes situationnels et cognitifs ayant pour enjeux les relations et les interactions des sujets parlants » (Galatanu, 2010 : 125), nous permettra de reconstruire, à travers les déploiements argumentatifs de Robin Gecht, l'identité régénérée, normale, qu'il véhicule dans et par son discours. Pour ce faire, nous partons dans notre analyse de certaines hypothèses avancées dans la perspective de la Sémantique des possibles argumentatifs (Galatanu, 1999 ; 2000 ; 2007a \& b) concernant la construction des identités dans le discours. À travers ces hypothèses, Galatanu (2010 : 125) conçoit l'identité comme « une 
construction à travers le sens produit dans et par le discours, d'images de soi pour soi et/ou pour autrui, d'autrui pour soi et/ou pour autrui ». Les deux hypothèses sous-jacentes à notre recherche, sont formulées ainsi par Galatanu :

« la restauration identitaire, caractérisée par la mobilisation de valeurs axiologiques positives, sera marquée par la mobilisation consciente, voire volontaire ou même stratégique ou, inversement, involontaire, de dénominations des actes sanctionnés moins négatives [...], et de dénominations à valeur plutôt aléthique ou déontique pour parler de la causalité de cet acte.

Lorsque des mots à valeurs négatives fortes sont mobilisés, leurs déploiements argumentatifs s'accompagnent d'une flexion de polarité discursive ou d'une flexion de polarité discursive et sémantique » (Galatanu, 2009 : 129).

Pour suivre la reconstruction de cette identité discursive régénérée à travers le discours de Robin Gecht, nous avons étudié les onze lettres envoyées par Robin Gecht à sa correspondante Jennifer Furio. Nous nous limitons dans notre analyse à deux éléments qui nous paraissent majeurs dans cette dynamique discursive identitaire, à savoir l'acte et l'acteur criminels. Ceuxci nous permettront de reconstruire l'univers sémantico-discursif de Robin Gecht.

Nous identifierons dans un premier temps les opérations énonciatives de dénomination, de désignation, de qualification, voire de discrimination relatives à l'acte criminel, ces opérations étant, pour nous, des Déclarations instituant une identité posée ou supposée par Robin Gecht face à une autre imposée par le monde médiatique et judiciaire et acceptée par les autres.

Dans un second temps, et par rapport à l'acte criminel et sa désignation par Robin Gecht, nous analyserons la dynamique discursive de « restauration identitaire » de l'acteur Robin Gecht, une dynamique qui se profile tout en véhiculant un ensemble de valeurs s'orientant toutes vers une régénération identitaire.

\section{L’univers sémantico-discursif de Robin à travers son acte}

Contrairement aux trois autres membres de la bande de « Chicago Rippers », Robin Gecht, le présumé chef de la bande, nie tout et clame son innocence ; il affirme : "First mistake...is considering me a serial killer... am not considered one... I have never killed or took part in any such acts nor ever charged in any murders of anyone » (cité par Furio, 1998). Donc placé face à une identité qu'on lui impose, voire un ethos préalable ou prédiscursif, Robin s'en empare pour chercher à s'en affranchir, à réinventer son ethos qui, rappelle Amossy (2012: 110), est lié à « la construction d'une identité qui permet en même temps de créer un rapport nouveau à soi et à l'autre ». Cette construction identitaire transparaît à travers les différentes qualifications pour désigner l'acte criminel, fournies dans le discours par Robin Gecht. 


\begin{tabular}{|c|l|l|}
\hline Désignation de l'acte & Fréquence \% & \multicolumn{1}{|c|}{ Exemples } \\
\hline $\begin{array}{c}\text { Désignations à valeur épisté- } \\
\text { mique (exclu, incertain) }\end{array}$ & $73,33 \%$ & $\begin{array}{l}\text { - Fantasy, fiction, lies, sick rumors, nonsense } \\
\text { - Bullshit, all kinds of shit, trash, sensationalistic bullshit }\end{array}$ \\
\hline $\begin{array}{c}\text { Désignations à valeur morale } \\
\text { négative }\end{array}$ & $10 \%$ & $\begin{array}{l}\text { Ill things, framing (au sens de « monter un coup } \\
\text { contre quelqu'un ») }\end{array}$ \\
\hline $\begin{array}{c}\text { Désignations à valeur morale } \\
\text { négative et déontique juridique }\end{array}$ & $10 \%$ & Murder, crime(s), rape charge \\
\hline $\begin{array}{c}\text { Désignations à valeur axiolo- } \\
\text { gique affective }\end{array}$ & $6,66 \%$ & Horror \\
\hline
\end{tabular}

Tableau 1. Désignations de l'acte criminel par Robin Gecht

Comme le résume le tableau 1, ce sont des désignations essentiellement chargées de valeurs épistémiques qui relèvent de l'exclu eu égard à l'identité que revendique Robin. Pour lui, tout s'inscrit dans une logique fictionnelle, imaginaire qu'a voulu lui « coller sur le dos », selon lui, le système judiciaire et médiatique. L'opposition qu'il établit, nous le verrons, tout le long de ces lettres entre les absurdités (Bullshit) et les faits (Facts) accentue la distinction entre la nouvelle image qu'il cherche à faire reconnaître par l'institution extérieure, par autrui à travers sa correspondante, et l'image d'un criminel imposée de fait par le Système. La discrimination entre ces deux images se voit aussi renforcée par les désignations à valeur morale négative que les autres veulent lui attribuer. II disait dans sa deuxième lettre, en parlant des médias, que: "Better and more ill-things said about Mr. Gecht, the better it sells ». D'après lui, l'acte criminel, sans qu'il le nomme, est un coup monté par la police contre lui, lui-même se présentant comme « a good person at heart ». Même lorsqu'il parle de crime (The State's Attorney who tell them about this crime), il ne s'approprie pas le mot; c'est en l'attribuant au Système juridique qu'il l'évoque pour maintenir davantage cette dynamique de discrimination entre les deux images : celle du criminel voulue par les autres et celle d'un individu normal comme les autres. Dans «How can this Rape charge [for which I got] 60 yrs stand without supporting evidence?, une autre désignation de ses crimes, à savoir Rape charge, apparaît également dans un contexte de remise en cause de son inculpation par le système judiciaire, qui lui refuse en même temps une analyse ADN. Dans / have never killed or took part in any such acts nor ever charged in any murders of anyone, la désignation par meurtre est également récusée.

Enfin, cette dynamique d'opposition identitaire, voire de discrimination, à travers la désignation de l'acte criminel se voit confirmée dans la dixième lettre de Robin Gecht lorsqu'il qualifie les actes des trois autres membres de la bande de « Chicago Rippers » d' « horreur » : " there had to be signs that I didn't or may be refused to see in order to stop their horror "; "But to involve me in their horror was way beyond any belief till it happened».

Pour résumer cette première partie sur la dynamique de restauration identitaire de Robin Gecht au travers de la désignation de l'acte criminel, nous dirions que celle-ci s'est établie sur quatre plans:

- $\quad$ épistémique, où Robin oppose sa vérité aux absurdités des autres;

- moral, où il cherche à montrer qu'il est en dehors de toute immoralité, contrairement aux autres qui médisent son image et complotent contre lui ;

- moral et déontique, auquel il fait appel pour confirmer l'opposition entre lui-même et le système judiciaire ; 
- axiologique affectif, à travers lequel il redore et renouvelle son image ; cela est confirmé, entre autres, par sa déclaration : «I too love life and family, I can hurt, feel remorse, and cry as you do. [...] I never learned to hate, to want to take another human life without cause ». À travers cette déclaration, Robin Gecht se crée une existence nouvelle, autre que celle véhiculée par les autres représentés par les médias, mais qui se veut tout de même identique à « certains autres », identifiés en l'image de sa correspondante, Jennifer Furio.

Qu'en est-il de cette reconstruction identitaire relative à l'image de l'acteur plutôt que de son acte?

\section{L'univers sémantico-discursif de Robin à travers son image d'acteur}

Robin Gecht, à travers ses lettres, établit une opposition assez nette entre lui - la victime - et certains autres, comme les trois membres de la bande de « Chicago Rippers » ou le système judiciaire et médiatique. Cette opposition sert une dynamique identitaire de restauration, renforcée par une sous-dynamique de désaveu et de contestation. Cette dynamique de désaveu se voit portée par un ensemble de valeurs véhiculé par le discours d'échange épistolaire, lui aussi hors-normes.

Parmi ces valeurs, les valeurs éthiques et morales, en l'occurrence celles liées à la croyance religieuse, sont très présentes. Le tableau 2 résume, à partir de la signification des mots ou du sens générés dans le discours, cette opposition entre les déclarations à valeurs religieuses d'affirmation/restauration et celles sous-tendues par des valeurs éthiques et morales de désaveu. 


\begin{tabular}{|c|c|}
\hline $\begin{array}{c}\text { Déclarations à valeurs éthiques/morales } \\
\text { d'affirmation }\end{array}$ & $\begin{array}{c}\text { Déclarations à valeurs éthiques/morales de } \\
\text { désaveu }\end{array}$ \\
\hline $\begin{array}{l}\text { Faith and God keeps me fighting } \\
\text { It was a warning from above saying } \\
\text { I have learned to forgive } \\
\text { Fair justice; pray for justice } \\
\text { Please forgive me for not having faith or trust } \\
\text { in most these days } \\
\text { By having faith and trust } \\
\text { As God intended for us } \\
\text { Justice } \\
\text { I too love life and family I can hurt, and feel } \\
\text { remorse } \\
\text { I never learned to hate } \\
\underline{\text { Thank God }} \\
\text { I do know } \underline{\text { meaning of sacrifice well }} \\
\text { May be God will forgive them } \\
\underline{\text { God sends me back to HELL }} \\
\underline{\text { JUSTICE FOR YOU AT ITS BEST }} \\
\underline{\text { My question often to God }} \\
\text { Much more in showing love and concern for } \\
\underline{\text { others }} \\
\text { I'll then get fair justice as deserved } \\
\text { God teaches us to love and be kind }\end{array}$ & $\begin{array}{l}\text { Everyone will have their day in Court } \\
\text { It would be a great injusice to consider me if } \\
\text { you wrote and considered me as such } \\
\text { With it [money] you get fair justice } \\
\text { Cases that are unfair and injust } \\
\underline{\text { No justice }} \\
\text { I don't only face the injustices } \\
\text { I could never live with killing or knowing I was } \\
\underline{\text { responsible for taking one's life }} \\
\text { I would never dream of hurting another hu- } \\
\text { man being }\end{array}$ \\
\hline
\end{tabular}

Tableau 2. Les valeurs éthiques et morales véhiculées par les déclarations de Robin Gecht

Ce tableau, de par les valeurs qu'il fait apparaître, permet d'identifier cette double dynamique identitaire oscillant entre contestation d'une image immorale qu'on a voulu, selon lui, associer à sa personne sans justice avérée, et affirmation d'une moralité qui pour le moins s'avèrerait identique à celle de toute autre personne morale. Cette dialectique identitaire qui transparait à travers les lettres de Robin Gecht est symp- 
tomatique d'une volonté d'affirmer une image de soi pour soi, mais surtout pour autrui, et de l'autre aussi pour se distinguer soi-même.

De ce discours hors-normes émerge également un champ sémantique portant des valeurs épistémiques venant appuyer les valeurs éthiques et morales présentes, encore une fois dans une double dynamique, à la fois pour désavouer les mensonges des autres et affirmer la vérité de soi. Robin Gecht se présente à travers son discours comme un homme de vérité. Le tableau 3 illustre cette image d'homme détenteur de la vérité, soucieux qu'elle soit dite.

\begin{tabular}{|c|c|}
\hline $\begin{array}{l}\text { Déclarations à valeurs épistémiques pour } \\
\text { soi }\end{array}$ & Déclarations à valeurs épistémiques pour l'autre \\
\hline As truthful as possible & Lies will get us nowhere \\
\hline NO, NOT AN OPINION, AFACT & Hiding the truth \\
\hline I can divide facts from fiction & Just wish he'd tell the truth as to myself \\
\hline It mattered to you had the truth & Far from facts or any truth \\
\hline I'll not stop with the truth & If truth is used not fiction \\
\hline \multicolumn{2}{|l|}{ Digging for truth } \\
\hline \multicolumn{2}{|l|}{ Is important here as to truth } \\
\hline \multicolumn{2}{|l|}{ Truth or fact } \\
\hline \multicolumn{2}{|l|}{ To give you truth } \\
\hline \multicolumn{2}{|l|}{ That is truth and fact } \\
\hline Help me with the truth & \\
\hline
\end{tabular}

Tableau 3. Les valeurs épistémiques véhiculées par les déclarations de Robin Gecht

Ces différentes déclarations constituent pour leur auteur une sorte de recréation identitaire à travers la reconnaissance d'une fonction-statut de l'identité honnête et véridique et, en même temps, occultent la fonction-statut qui lui a été initialement associée, celle du «tueur en série », du «monstre ». Robin Gecht avance une image de soi, homme de vérité, opposée à une image d’autrui, homme de mensonge.

D’autres valeurs, moins présentes que les deux premières, cette fois-ci de nature axiologique affective, viennent aussi participer à ce renouveau identitaire, non pas dans une logique d'opposition ou de discrimination, mais dans une optique d'identification à l'individu normal qui peut exprimer des émotions. Robin Gecht n'est pas seulement l'homme croyant et moral, l'homme de vérité, mais aussi l'homme qui a des sentiments, qui se soucie des autres, qui les aime, qui peut souffrir, pleurer; bref qui est normal. Le tableau 4 présente quelques passages où Robin Gecht affirme être un homme de sentiments, un homme comme les autres. 


\begin{tabular}{l} 
Déclarations à valeurs axiologiques affectives \\
\hline You have no idea the pain and hurt I face and feel every single day \\
Bhat might give you an idea of what's is in my heart as to feelings \\
An insight of the love, NOT HATE that's within \\
Ilove people \\
I had tears in my eyes \\
Heart warming I must say \\
Another true and honest feeling I hide from most here. They in prison call it weakness
\end{tabular}

Tableau 4. Les valeurs axiologiques affectives véhiculées par les déclarations de Robin Gecht

Enfin, ce renouveau identitaire déclaré par Robin Gecht à travers ses lettres est revêtu d'une sous-dynamique de victimisation où il refuse d'accepter qu'on le désigne comme « tueur en série ». Il affirme à maintes reprises (cf. tableau 5) qu'il est une victime et non pas un monstre contrairement à ce qu'on a voulu faire croire. Cette sous-dynamique fait apparaître une volonté de Robin Gecht de se défaire de cette image hors-normes qui lui est associée comme tueur en série, afin de se reconstruire sa nouvelle image, pour lui et pour les autres.

Déploiements argumentatifs de victimisation
I'm not considered one [serial killer]
An injustice to consider me as such
I too became a victim
Stop using the term SERIAL KILLER towards me
I hurt so much just hearing that word and all the lies I hear and read yet no one has taken time to know me,
but first to judge me.
I'm ill of the concept
The monster they portray I am
I feel I'm a victim here too
Does that make me a monster?

Tableau 5. Les déploiements argumentatifs de victimisation de Robin Gecht

Et pour contrer ces qualifications, Robin Gecht choisit de les démentir en s'affirmant comme une personne normale. Si l'on considère les déploiements argumentatifs suivants disséminés dans son discours: 
1. I never killed anyone and I love woman

2. Ilove woman

3. I love and have a lot of respect for them

4. I was never involved there nor in Chicago

5. I was tested and cleared in du-Page Co

6. I'm a good person at heart

Robin Gecht semble développer le schéma argumentatif suivant:

+ Amour pour la femme DONC - Tueur en série DONC Acquittement DONC Personne intègre

Il affirme par ailleurs que son obsession pour les seins n'est ni plus ni moins l'obsession de tout homme " normal »: "I have no real obsession with breast in that form. Only a very sick person would even think that »; et estime que ce que l'on dit sur lui n'est qu'absurdités: "As to shit you're reading about breast being removed or having sex with breast BULLSHIT».

Nous pouvons ainsi dire que les déploiements argumentatifs de Robin Gecht, sous forme de déclarations (au sens de Searle), liés à son acte et à sa personne, ont permis de créer une réalité par le simple fait de la représenter comme existante. C'est la réalité d'une identité autre que celle représentée, comme existante, par les autres, au moyen d'autres discours judiciaires ou médiatiques. Robin Gecht a cherché par le biais de ses lettres, par cet échange épistolaire, à se recréer une identité normale et à la faire reconnaître par l'autre, à lui octroyer une intentionnalité collective (Searle, 2010), par l'intermédiaire de Jennifer Furio, sa correspondante. Cette nouvelle identité est affirmée et réaffirmée par une dynamique de restauration, sous-tendue par une double sous-dynamique, à la fois de désaveu et d'affirmation, l'ensemble corroboré par une image de victimisation qui s'accentue par la remise en cause de son inculpation par le système judiciaire, qui lui refuse sa demande répétitive d'une analyse ADN.

\section{Conclusion}

Nous avons essayé à travers cette reconstruction de l'univers discursif d'un tueur en série, du point de vue de l'identité qu'il entend déclarer et des actes qu'il revendique, de faire apparaître les dynamiques identitaires qui sous-tendent sa volonté de régénérer son image, face à celle que l'on a construite de lui, judiciairement et médiatiquement. Robin Gecht développe, dans les lettres envoyées à Jennifer Furio, toute une panoplie de mécanismes sémantico-discursifs véhiculant en même temps un ensemble de valeurs, oscillant entre valeurs épistémiques, morales, déontiques et axiologiques, qui lui permettent de se créer une existence nouvelle, une identité épistémiquement subjective (Searle, 2010), dans la mesure où cette nouvelle identité revendiquée a besoin d'être reconnue pour exister. C'est donc tout l'apport de cet échange épistolaire, du moins aux yeux de Robin Gecht, qui cherche à se faire reconnaître une identité par les autres, représentés ici par sa correspondante Jennifer Furio. Ses lettres constituent chacune un ensemble de déclarations, voire sont toutes une seule Déclaration lui conférant une fonction-statut de « personne normale » par opposition à la fonction-statut de «personne hors-normes » ou « anormale » qu'on a voulu lui attribuer au moyen de déclarations judiciaires et médiatiques. 


\section{Bibliographie}

Amossy Ruth (2012), L'argumentation dans le discours, Paris, Armand Colin.

Barbier Jean-Marie et Galatanu Olga (1998), « De quelques liens entre action, affects et transformation de soi », J.-M. Barbier et O. Galatanu (dir.), Action, affects et transformation de soi, Paris, Puf, 45-70.

Furio Jennifer (1998), The Serial Killer Letters: A Penetrating Look Into the Minds of Murderers, Philadelphia, The Charles Press Publishers.

Galatanu Olga (1999), «Argumentation et analyse du discours », Y. Gambier et E. Suomela-Salmi (dir.), Jalons 2, Turku, Université de Turku, 41-54.

Galatanu Olga (2000), «Langue, Discours et systèmes de valeurs », dans Eija Suomela- Salmi (dir.), Curiosités linguistiques, Turku, Université de Turku, 80-102.

Galatanu Olga (2007a), «Pour une approche sémantico-discursive du stéréotypage à l'interface de la sémantique théorique et de l'analyse du discours », dans Henri Boyer (dir.), Stéréotypage, stéréotypes : fonctionnements ordinaires et mises en scène, tome 4 : Langue(s), Discours, Paris, L'Harmattan, 89-100.

Galatanu Olga (2007b), "Sémantique des possibles argumentatifs et axiologisation discursive », dans D. Bouchard, I. Evrard et E. Vocaj (dir.), Représentations du sens linguistique II, Louvain-la-Neuve, De Boeck-Duculot, 313-325.

Galatanu Olga (2009), «Les incidences sémantiques des déploiements argumentatifs dépendants du co-(n) texte de production du discours », E. Havu, J. Härmä, M. Helkkula, M. Larjavaara et U. Tuomarla (dir.), La langue en contexte. Actes du colloque "Représentations du sens linguistique IV », Helsinki 2830 mai 2008, Mémoires de la Société Néophilologique d’Helsinki, tome LXXVIII, Helsinki, Société Néophilologique, 391404.

Galatanu Olga (2010), «Pour une approche sémantico-discursive du concept d'identité : faute, crime et dynamique discursive », M. Palander-Collin, et al. (dir.), Constructing Identity in Interpersonal Communication/Construction identitaire dans la communication interpersonnelle, Mémoires de la Société Néophilologique de Helsinki, tome LXXXI, Helsinki, Société Néophilologique, 125-138.

Monnet Eric et Navarro Pierre (2009), « Les institutions sont-elles dans la tête ? Entretien avec John Searle », Tracés. Revue de Sciences humaines [En ligne], 17, mis en ligne le 01/11/2011, consulté le 05 juin 2015. http://traces.revues.org/4270

Searle John Rogers (2010), Making the Social World: The Structure of Human Civilization, Oxford, Oxford University Press. 